\title{
ADSORÇÃO DE ÁCIDOS HÚMICOS EM LATOSSOLO ROXO NATURAL E TRATADO COM OXALATO DE AMÔNIO(1)
}

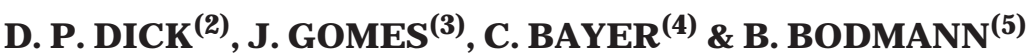

\begin{abstract}
RESUMO
A interação de substâncias húmicas com minerais no solo pode influir na dinâmica da decomposi ção da matéria orgânica e na sua estabilidade. A adsorção de dois ácidos húmicos em Latossolo Roxo natural (LR natural) e tratado com oxalato de amônio (LR tratado) foi efetuada, com vistas em investigar os mecanismos de adsorção e os principais grupos funcionais envolvidos, bem como relacionar a capacidade adsortiva de acordo com as características químicas e moleculares dos adsorbatos. Os ácidos húmicos extraídos com solução de $\mathrm{NaOH}$ 0,5 $\mathrm{N}$ de uma amostra de carvão (AHc) e de um solo Brunizém (AHs) foram caracterizados por análise elementar e espectroscopia de ${ }^{13} \mathrm{C}-\mathrm{R}$ MN. A parametrização dos dados experimentais das isotermas de adsorção foi do tipo y = A tanh Bx, e o modelo de Langmuir também foi aplicado. As amostras adsorvidas foram analisadas por espectroscopia no Infra-Vermelho por Reflectância Difusa com Transformada de Fourier (DRIFT). A adsorção dos ácidos húmicos no LR natural foi de natureza química, sendo evidenciado por DRIFT que a reação de troca de ligantes das carboxilas com a superfície dos minerais foi um mecanismo importante. $\mathrm{O}$ AHc, que apresentou caráter aromático mais elevado e maior teor de carboxilas na molécula, foi adsorvido em maior quantidade do que o AHs nos dois adsorventes, o que indica a possível participação de estruturas aromáticas na interação organomineral. No LR tratado, cuja área superficial específica foi superior (ASE $=140,9 \mathrm{~m}^{2} \mathrm{~g}^{-1}$ ), a adsorção foi mais elevada do que no LR natural (ASE $=66,1 \mathrm{~m}^{2} \mathrm{~g}^{-1}$ ). A maior ASE no LR tratado foi relacionada com o rompimento de microagregados no tratamento com oxalato de amônio que extraiu a porção cimentante dos óxidos de ferro que unia os minerais. A interação neste tipo de adsorvente, estimada segundo a equação $y=A$ tanh $B x$, foi mais forte do que na amostra natural, o que
\end{abstract}

(1) Trabalho apresentado em parte no VI Encontro de Química da Região Sul em Maringá (PR), de 4-6 de novembro de 1998. Recebido para publicação em maio de 1999 e aprovado em fevereiro de 2000.

(2) Professora Adjunta do Departamento de Físico-Química, Instituto de Química, Universidade Federal do Rio Grande do Sul (UFRGS). Av. Bento Gonçalves 9500, CEP 91501-970 Porto Alegre (RS). Professora PPG-Ciência do Solo, Faculdade de Agronomia, UFRGS. Bolsista do CNPq. E-mail: dpdick@if1.if.ufrgs.br.

(3) Graduanda do curso de Bacharelado em Química, UFRGS. Bolsista de IC do CNPq.

(4) Professor Adjunto do Departamento de Solos, UDESC, Caixa Postal 281, CEP 88520-000 Lages (SC).

(5) Professor Titular do Centro de Ciências Exatas e Tecnológicas, UNISINOS, CEP 93022-000 São Leopoldo (RS). 
pode estar relacionado com a presença de sítios de adsorção hidrofóbica pela presença do oxalato na superfície, com a exposição de sítios mais reativos após tratamento e pH mais ácido. 0 modelo de Langmuir mostrou-se adequado para explicar a adsorção na amostra natural, enquanto, no LR tratado com oxalato de amônio, o ajuste não foi satisfatório. Em geral, a aplicação da equação y =A tanh Bx forneceu melhor ajuste ( $R^{2}$ maior) do que a equação de Langmuir.

Termos de indexação: parametrização, troca de ligantes, ${ }^{13} \mathrm{C}-\mathrm{RMN}, \mathrm{DRIFT}$, interação química.

\title{
SUMMARY: ADSORPTION OF HUMIC ACIDS ON NATURAL AND AMMONIA- OXALATE TREATED OXISOL
}

\begin{abstract}
Theadsorption of two humic acids (HA) was carried out on a natural Oxisol (natural $L R$ ) and on an ammonium-oxalate treated sample (treated LR), in order to investigate the adsorption mechanisms and the main functional groups involved and relatetheadsorptive capacity with thechemical and molecular characteristics of theadsorbates. TheHA extracted with $0.5 \mathrm{~N} \mathrm{NaOH}$ solution from a coal sample (HAc) and from a Mollisol sample (HAs) werecharacterized by elemental analysis and ${ }^{13} \mathrm{C}-\mathrm{N}$ MR spectroscopy. Theexperimental data were fitted according to the equation $\mathrm{y}=\mathrm{A}$ tanh $\mathrm{Bx}$ and the model of Langmuir was also applied. Theadsorbed samples were analyzed by Diffuse Reflectance F ourier Transformed I nfrared Spectroscopy (DRIFT). The HA adsorption on the natural LR was of a chemical nature, with the ligand exchange reaction between the carboxyl groups and the mineral surfaces being an important mechanism, as shown by DRIFT. TheAHc had a higher aromatic character and a higher carboxyl content in its mol eculeand was adsorbed in greater quantity than the AH s in both adsorbents. This result al so suggests a possiblepartici pation of aromatic moieties in the organo-mineral interaction. The treated LR, which had a higher specific surface area (SSA $=140.9 \mathrm{~m}^{2} \mathrm{~g}^{-1}$ ), showed a greater adsorptive capacity than the natural LR (SSA $\left.=66.1 \mathrm{~m}^{2} \mathrm{~g}^{-1}\right)$. Thehigher SSA in thetreated samplewas related to the breakup of microaggregates by the oxal atetreatment, caused by theextraction of thecementing portion of iron oxides that bonded theparticles together. Theinteraction in this adsorbent, as estimated by the parametrization $\mathrm{y}=\mathrm{A} \tanh \mathrm{Bx}$, showed higher chemical affinity than in thenatural sample. This can be related to the adsorbed oxal ate that created hydrophobic adsorption sites, the exposure of more reactive sites after the treatment and the lower $\mathrm{pH}$ value. The Langmuir model was adequateto explain theadsorption in thenatural sample, whilein the treated sample, the results obtained were not satisfactory. Generally, the proposed parametrization $y=A$ tanh $B x$ yielded the better fit (i.e. higher $R^{2}$ ) in comparison to the Langmuir equation.
\end{abstract}

Index terms: parametrization, ligand exchange, ${ }^{13} \mathrm{C}-\mathrm{NMR}, \mathrm{DRIFT}$, chemical interaction.

\section{NTRODUÇÃO}

A interação das substâncias húmicas com os minerais nosolo éresponsável, em grandeparte, pela sua estabilidade à decomposição microbiana (Duxbury et al., 1989, Bayer, 1996) e pela estabilidade deagregados (Muneer \& Oades, 1989). Estes compostos orgâni cos demédia a el evada massa molecular são constituídos por estruturas al ifáticas earomáticas, às quais estãoligados os mais variados grupos funcionais (Stevenson, 1994), o que possibilita inúmeras formas de interação com as superfícies minerais, cuja atuação concomitanteresulta em alta energia de ligação total.
A interação das substâncias húmicas com superfícies hidroxiladas tem sido freqüentemente evidenciada por meio de testes de adsorção, em que o mecanismo de adsorção preponderante é a troca de ligantes, seguido por interações tipo pontes de hidrogênio e pontes catiônicas (Parfitt et al., 1977; Tipping, 1981; Morelli \& Bortoluzzi, 1992; Gu et al., 1994; Varadachari et al., 1997). Também a análise por infravermel ho da matéria orgânica adsorvida na superfície oxídica tem fornecido informações sobre os grupos funcionais envol vidos no processo (Parfitt et al., 1977; Ristori et al., 1992; Gu et al., 1994; Kaiser $\&$ Zech, 1997a).

Em superfícies de goethita, os sítios de coordenação são as hidroxilas mono-coor denadas da face (100) e, 
na gibbsita, são as bordas laterais (Parfitt et al., 1977). Foi observado também que as hidroxilas bicoordenadas das faces (001) da gibbsita adsorveram, por pontes de hidrogênio, compostos húmicos na forma não ionizada. Varadachari et al. (1997) verificaram em óxidos sintéticos que a forma de interação de ácidos húmicos com a superfície da goethita e da hematita pode ser tanto por meio de pontes de cátions como por meio de reações de coordenação diretamente nos sítios hidroxilados, tendendo as ligações a ser mais fortes na hematita.

Schulthess \& Huang (1991) observaram que a adsorção em sítios Al-OH deóxidos de alumínio e de caulinita (faces laterais) ocorreu num amplo intervalo de $\mathrm{pH}$ (2 a 10), o que é indicativo de uma forte interação. Em sítios Si-OH de óxido de silício e de montmorilonita, a adsorção foi rel evante apenas em níveis ácidos de $\mathrm{pH}(<3)$, devido provavel mente ao estado protonado das substâncias húmicas. Nestes tipos de superfícies, a adsorção acentuou-se quando as substâncias húmicas foram adicionadas na forma de sais de níquel, ficando evidenciado o mecanismo de pontes catiônicas.

Fontes (1990) verificou que o complexo matéria orgânica-goethita era muito estável e diminuiu a adsorção de fosfato pela goethita, enquanto cristais de goethita livre de matéria orgânica apresentaram alta capacidade de fixação de fosfato. Pela análise no infravermelho, constatou também quea interação com a superfície era do tipo troca de ligantes.

Vários model os matemáticos têm sido aplicados a dados experimentais para descrever a adsorção em solos (Sparks, 1995). Os modelos mais comumente empregados são os de Freundlich e de Langmuir, que, apesar de suas limitações quanto à fundamentação teórica (Sposito, 1989), descrevem freqüentemente, de maneira satisfatória, a adsorção de ânions em sol os (Olsen \& Watanabe, 1957; Dick, 1986; Gu et al., 1994; Valladares et al., 1998). O modelo de Langmuir, inicialmentedesenvolvido para descrever a adsorção de moléculas gasosas em superfícies planas, assume a adsorção em monocamada em uma superfície uniforme e permite estimar a capacidade de adsorção máxima (Stevenson, 1994; Sparks, 1995). Em solos, este modelo geralmente fornece bons resultados para testes de adsorção envolvendo ânions que apresentem alta afinidade pela superfícieadsorvente (Dick, 1986; Gu et al., 1994; Wang et al., 1997).

Os objetivos principais deste estudo foram avaliar a adsorção de dois ácidos húmicos em L atossolo Roxo natural por meio da aplicação do modelo de Langmuir e da parametrização $\mathrm{y}=\mathrm{A} \tanh \mathrm{Bx}$ a dados experimentais; inferir sobre mecanismos de adsorção e principais grupos funcionais envolvidos por meio de espectroscopia de infravermelho e relacionar a capacidade adsortiva de acordo com as características químicas e moleculares dos adsorbatos. Visando avaliar o efeito da remoção de óxi dos de ferro pobremente cristal inos na capacidade adsortiva do sol o, foram também real izados testes de adsorção em amostra tratada com oxalato deamônio.

\section{MATERIAL E MÉTODOS}

O adsorvente empregado foi a fração $<53 \mu \mathrm{m}$ da camada de $0-2,5 \mathrm{~cm}$ do horizonte $A$ de um $L$ atossolo Roxo distrófico ( $\mathrm{LRd}$, unidade de mapeamento Santo Ângelo, RS), obtida por peneiramento úmido após agitação horizontal do solo (fração terra fina), durante 15 horas, com duas esferas de vidro. Os principais constituintes minerais do adsorvente, determinados por Bayer (1996), foram os óxidos de ferro pouco cristalinos $\left(5,99 \mathrm{~g} \mathrm{~kg}^{-1}\right)$, óxidos de ferro cristalinos $\left(233,1 \mathrm{~g} \mathrm{~kg}^{-1}\right)$, caulinita $\left(610 \mathrm{~g} \mathrm{~kg}^{-1}\right) \mathrm{e}$ quartzo (aproximadamente $150 \mathrm{~g} \mathrm{~kg}^{-1}$ ). Os adsorbatos estudados foram ácido húmico extraído de um solo Brunizém (AHs) (Rio Pardo, RS) e ácido húmico extraído de uma amostra de carvão (AHc) da mina de Seival, Candiota (RS).

\section{Preparação dos adsorventes}

Os testes de adsorção foram realizados em amostra natural e em amostra tratada com oxalato de amônio a pH 3 (Schwertmann, 1964). Ambos os adsorventes foram tratados com $\mathrm{H}_{2} \mathrm{O}_{2}$ p.a. $95 \%$, à temperatura de 30-35C, para remoção da matéria orgânica original e de oxalato, atéque não houvesse mais reação com a adi ção de $\mathrm{H}_{2} \mathrm{O}_{2}$. As amostras foram secas a $60^{\circ} \mathrm{C}$ etrituradas em gral deágata. $\mathrm{O} \mathrm{pH}$ em água foi determinado na amostra natural e na amostra tratada com oxalato de amônio, empregando-se $10 \mathrm{~g}$ de solo e $25 \mathrm{ml}$ de solução (EMBRAPA, 1997).

\section{Preparação das soluções de adsorbatos}

Os $\mathrm{AH}$ foram obtidos por extração alcalina $(3 \mathrm{~g}$ de carvão e $10 \mathrm{~g}$ de sol o; $30 \mathrm{ml}$ de sol ução de $\mathrm{NaOH}$ $0,5 \mathrm{~N}, 3$ vezes $2 \mathrm{~h}$ de agitação), precedida por tratamentocom $\mathrm{HCl} 0,1 \mathrm{~N}$ (30 ml, 3 vezes $2 \mathrm{~h}$ ), epurificados com solução de $\mathrm{HF}-\mathrm{HCl} 5 / 5 \%$ (3 vezes $2 \mathrm{~h}$ ), segundo método adaptado e descrito por Dick et al. (1998).

A solução matriz de $\mathrm{AH}$ foi preparada, solubilizando-se uma amostra purificada úmida com solução de $\mathrm{NaCl}$ 0,1 M e de $\mathrm{NaOH}$ 0,01 M, até obterse uma solução a pH 7 e I =0,05 M com volume conhecido. A concentração de $C$ em solução foi determinada pel o método Walkley \& Black, descrito por Tedesco et al. (1995). A partir desta análise e do resultado da análise el ementar (descrito mais adiante), calculou-se a concentração de $\mathrm{AH}$ em solução (mg AH L-1). As soluções deadsorbato foram preparadas a partir da solução matriz por diluição, abrangendo um intervalo de concentração de aproximadamente 0 a $685 \mathrm{mg} \mathrm{AH} \mathrm{L-1,} \mathrm{para} \mathrm{oAHs,} \mathrm{e}$ de 0 a $714 \mathrm{mg} \mathrm{AH} \mathrm{L-1,} \mathrm{para} \mathrm{o} \mathrm{AHc,} \mathrm{ajustando-se} \mathrm{o}$ $\mathrm{pH}$ a 7 el $=0,05 \mathrm{M}$. 


\section{Caracterização química e espectroscopia de ressonância magnética nuclear do ${ }^{13} \mathrm{C}$ (13C- RMN) dos adsor batos e determinação da área superficial específica (ASE) dos adsorventes}

Para caracterização dos adsorvatos, os $\mathrm{AH}$ foram extraídos, purificados e secos a $60^{\circ} \mathrm{C}$ em estufa a vácuo. Os teores de $\mathrm{C}, \mathrm{H}$ e $\mathrm{N}$ foram determinados por análise el ementar (analisador el ementar Perkin EImer 4000). O teor de cinzas foi determinado por cal cinação $750^{\circ} \mathrm{C}$ durante $4 \mathrm{~h}$. Os valores de $\mathrm{C}, \mathrm{H}$ e $\mathrm{N}$ foram corrigidos para uma base livre de cinzas e o teor de oxigênio foi calculado por diferença de $1.000 \mathrm{~g} \mathrm{~kg}^{-1}$.

As características moleculares foram determinadas por ${ }^{13} \mathrm{C}-\mathrm{RMN}$ no estado líquido (Institut für Spektrochemie und Angewandte Spektrokopie, Dortmund, Alemanha). Amostras úmidas de $\mathrm{AH}$ purificados foram diluídas em NaOD- $\mathrm{D}_{2} \mathrm{O}$ a pD $=8,5$ até volume de $0,55 \mathrm{ml}$ e col ocadas em tubo de $5 \mathrm{~mm}$. O espectro foi obtido num espectrômetro J EOL GX 400, utilizando-se uma freqüência de $100 \mathrm{MHz}$ para ${ }^{13} \mathrm{C}$, intervalo de freqüência de 480 ppm, intervalo de repetição de pulso de 1,2 s, número de scans de $1.10^{5}$ a 2,15.105. As atribuições das bandas foram feitas segundo Skjemstad et al. (1996) eas proporções das estruturas que continham carbono na molécula de $\mathrm{AH}$ foram calculadas por integração das respectivas áreas. Calculou-se o fator de aromaticidade, fa, segundo Hatcher et al. (1981), que consistiu em dividir a área da banda dos grupos aromáticos (165 a 100 ppm) pela soma das áreas dos grupamentos al ifáticos (100-0 ppm) earomáticos.

A ASE dos adsorventes foi determinada com três repetições em amostras secas ao ar, usadas nos testes de adsor ção, pel o método da adsor ção de água a 20\% de umidade relativa (UR), segundo Quirk (1955). Cerca de 2,000 g desolo (exatamente pesadas) foram col ocados em frascos pesa-filtro de $8 \mathrm{~cm}$ de diâmetro e desidratados em dessecador sob $\mathrm{P}_{2} \mathrm{O}_{5}$, durante 10 dias, a $25^{\circ} \mathrm{C}$. Após pesagem da amostra desidratada, foi colocada no dessecador uma sol ução saturada deacetato de potássio ( $20 \%$ UR ) e osistema foi mantido fechado por mais 10 dias. A amostra foi novamente pesada. Considerou-se que a $20 \%$ UR a água e adsorvida na fase sólida numa camada monomolecular, ocupando cada molécula de água uma área de 0,108 $\mathrm{nm}^{2}$. Portanto, $1 \mathrm{mg}$ de água adsorvida a $20 \%$ UR corresponde a uma área de recobrimento de 3,6 $\mathrm{m}^{2}$ (Quirk, 1955).

\section{Determinação das isotermas de adsorção}

Para determinar as isotermas de adsorção, empregaram-se, em temperatura ambiente, as seguintes condições experimentais: $0,3 \mathrm{~g}$ de solo, $30 \mathrm{ml}$ de solução de $\mathrm{AH}$, 30 min de agitação seguidos de20 min derepouso. Após a adsorção, osobrenadante foi separado por centrifugação durante $10 \mathrm{~min}$ a 2.000 rpm, e a concentração de carbono em solução foi determinada pelo método espectrofotométrico, descrito em Dick et al., 1998. A curva padrãofoi feita com as soluções adsorventes adicionadas ao solo, cujas concentrações foram cal culadas, considerando a diluição a partir da solução matriz. A partir da concentração de $\mathrm{C}$ dos $\mathrm{AH}$, determinada por análise elementar, os resultados foram recalculados para mg AH L-1 e a quantidade adsorvida por diferença entre as concentrações (inicial e final).

As isotermas de adsorção foram traçadas, relacionando-se a quantidade adsorvida (Qads, mg AH g g $^{-1}$ solo) com a concentração em equilíbrio (Ceq, mg AH L-1).

Aos dados experimentais foi aplicada a equação $\mathrm{y}=\mathrm{A}$ tanh $\mathrm{Bx}$, obtida por regressão simples. Considerando que a tangente hiperbólica pode apresentar o val or máximo de 1 (um), resulta que o coeficiente A corresponderá à adsorção máxima (valor máximo para y). Tal parametrização permite também estimar a afinidade entre o adsorvente e o adsorvato, conforme descrito a seguir. A reação de adsor ção pode ser assim esquematizada:

[Sítios de superfície] $+\mathrm{AH}=[\mathrm{AH}$-Superfície]

Analisando a fase inicial das isotermas, em quea quantidadeadsorvida é muito inferior à quantidade máxima e a quantidade de sítios ativos abundante (atividade $=1$ ), verifica-se que a constante $K$ desta reação é diretamente proporcional ao quociente [quantidade de $\mathrm{AH}$ adsorvido]/[quantidade de $\mathrm{AH}$ em equilíbrio na solução]. Este quociente, por sua vez, pode ser calculado pela inclinação da parte inicial da isoterma de adsorção. Por outro lado, a constante $\mathrm{K}$ permite calcular a energia livre da reação por meio da equação:

$$
\Delta \mathrm{G}^{\circ}=-\mathrm{RT} \ln K
$$

A parteinicial da equação da tangentehiperbólica pode ser calculada, utilizando-se a expansão de Taylor $y=A B x+O^{3}(x)$, representando $\mathrm{O}^{3}(x)$ graus superiores $\geq 3$ em $x$. AB representa a inclinação próxima da origem e, nesta região, $\mathrm{O}^{3}(\mathrm{x})$ pode ser desprezado pois assume valores muito pequenos. Destemodo, oprodutodeA (normalizado para unidade de área) por B relaciona-se com a constante K.

Aos dados experimentais deadsorção foi ajustado também o model o de L angmuir na forma linearizada cuja equação é:

$$
C_{\text {eq }} / X=1 / K M+C_{e q} / M
$$

em que

$X$ = quantidade de $\mathrm{AH}$ adsorvida, $\mathrm{mg} \mathrm{AH} \mathrm{g}^{-1}$ solo;

$\mathrm{C}_{\text {eq }}=$ concentração de $\mathrm{AH}$ em equilíbrio, mg AH L-1;

M =adsorção máxima de AH estimada pel o modelo, mg AH g-1 solo;

$\mathrm{K}=$ coeficiente. 


\section{Espectroscopia no infravermelho dos adsor- ventes}

Amostras de adsorvente antes e depois da adsorção foram analisadas por espectroscopia na região do infravermel ho, por reflectância difusa com transformada de Fourier (DRIFT). O espectro foi determinado de 4.000 a $400 \mathrm{~cm}^{-1}$, com resolução de espectro de $4 \mathrm{~cm}^{-1}$ e acúmulo de 600 scans (espectrofotômetro BOMEM). Amostras que continham $\mathrm{AH}$ adsorvido foram preparadas, adicionando-se $30 \mathrm{ml}$ de solução concentrada de AHs (1,38 g AH L-1) edeAHc (3,50 g AH L-1) a $5 \mathrm{~g}$ de LR natural e de LR tratado com oxalato de amônio. Procedeu-se à agitação por 30 min, e a suspensão foi seca a $60^{\circ} \mathrm{C}$. A amostra seca foi lavada com água destilada até o sobrenadante ficar límpido e incolor, e o sol o com AH adsorvido foi seco em estufa a $105^{\circ} \mathrm{C}$. As amostras secas foram trituradas em gral deágata e armazenadas para análise. As atribuições das bandas deabsorção da matéria orgânica foram feitas com base em Baes \& Bloom (1989) e Kaiser et al. (1997), enquanto as da fração mineral, segundo Farmer (1974) e Delineau et al. (1994).

\section{RESULTADOS E DISCUSSÃO}

\section{Características químicas e moleculares dos ácidos húmicos}

O AHs apresentou teor de carbono inferior ao do $\mathrm{AHc}$, enquanto os teores de $\mathrm{H}$ e $\mathrm{N}$ foram superiores (Quadro 1). A alta razão $\mathrm{C} / \mathrm{N}$ obtida para o $\mathrm{AHC}$ refletea sua origem do carvão, pobre em N. NoAHs, a razão $\mathrm{H} / \mathrm{C}$ foi maior do que no $\mathrm{AHc}$, revelando mai or grau decondensação na amostra decarvão. As razões O/C foram semel hantes e este índice não permitiu diferenciar quimicamente as duas amostras.

No entanto, os resultados obtidos pela análise ${ }^{13} \mathrm{C}$ RMN permitiram distinguir as estruturas oxigenadas nas duas amostras: no $\mathrm{AHs}$, o oxigênio está principalmente em estruturas tipo celulose e
Quadro 1. Composição el ementar $\left(\mathrm{mg} \mathrm{g}^{-1} \mathrm{AH}\right) \mathrm{em}$ base livre de cinzas e razões $\mathrm{C} / \mathrm{N}, \mathrm{O} / \mathrm{C}$ e $\mathrm{H} / \mathrm{C}$ de ácido húmico de solo (AHs) e de carvão (AHc)

\begin{tabular}{cccccccc}
\hline Adsorbato & $\mathbf{C}$ & $\mathbf{H}$ & $\mathbf{N}$ & $\mathbf{O}^{(\mathbf{1})}$ & $\mathbf{C / N}$ & $\mathbf{O / C}(\mathbf{2})$ & $\mathbf{H} / \mathbf{C}^{(2)}$ \\
\hline AHs & 510 & 50 & 45 & 390 & 11 & 0,6 & 1,2 \\
AHC & 570 & 40 & 10 & 380 & 57 & 0,5 & 0,8
\end{tabular}

(1) obtido por diferença de $1.000 \mathrm{~g} \mathrm{~kg}^{-1}$. (2) razão atômica.

polissacarídeos (100-65 ppm), enquanto, no $\mathrm{AHc}$, o oxigênio está na forma de carboxilas (190-165 ppm) (Quadro 2). Os valores calculados do fator aromaticidade, fa, demonstram queo AH capresenta um caráter aromático superior ao do AHs, corroborando os resultados obtidos a partir da razão $\mathrm{H} / \mathrm{C}$. A maior abundância de estruturas tipo $\mathrm{CH}_{\mathrm{n}}-\mathrm{O}-$ $\mathrm{R}$ e a menor condensação no $\mathrm{AHs}$ refletem a sua origem mais recente, quando comparado com o AHc.

\section{Adsorção em L atossolo Roxo natural}

A representação gráfica das isotermas de adsorção no solo natural mostra que a adsorção máxima deAHs foi al cançada para as concentrações mais altas de adsorbato adicionado ao sistema, enquanto, para o AHc, os valores de quantidade adsorvida apenas tenderam para um nível de saturação(Figura 1). Para oAHs, a adsorção máxima estimada pela parametrização $y=A$ tanh $B x$ foi de $12,61 \mathrm{mg} \mathrm{AH} \mathrm{g}^{-1}$ solo $\left(\mathrm{R}^{2}=0,94\right)$ e, para o $\mathrm{AHc}$, foi de 25,94 mg AH g-1 solo $\left(R^{2}=0,80\right)$ (Quadro 3). Estes valores encontram-se dentro do intervalo de val ores já observados para óxidos de ferroe caulinita, puros ou sintéticos (Gu et al., 1994; Kaiser \& Zech, 1997a). Calculando os valores de adsorção máxima, A, por unidade de área (mg AH m-2), a partir dos dados de área superficial específica, obteve-se para o AHc aproximadamente o dobro do cal culado para o AHs (Quadro 3).

Quadro 2. Deslocamento químico, atribuições e intensidades relativas no espectro de ressonância magnética nuclear de ${ }^{13} \mathrm{C}$ e fator de aromaticidade (fa)

\begin{tabular}{cccccc}
\hline & \multicolumn{5}{c}{ Deslocamento químico (ppm) } \\
\cline { 2 - 5 } Adsorvato & $\begin{array}{c}\mathbf{1 9 0 - 1 6 5} \\
\text { C-carboxílico }\end{array}$ & $\begin{array}{c}\mathbf{1 6 5 - 1 0 0} \\
\text { C-aromático }\end{array}$ & $\begin{array}{c}\mathbf{1 0 0 - 6 5} \\
\mathbf{C H}_{\mathbf{n}}-\mathbf{0}-\mathbf{R}\end{array}$ & $\begin{array}{c}\mathbf{6 5 - 0} \\
\mathbf{C H}\end{array}$ & $\mathbf{f a}$ \\
\hline & & & & & \\
$\mathrm{AHS}$ & 5 & 57 & 20 & 18 & 0,60 \\
$\mathrm{AHC}$ & 14 & 65 & 5 & 16 & 0,76 \\
\hline
\end{tabular}




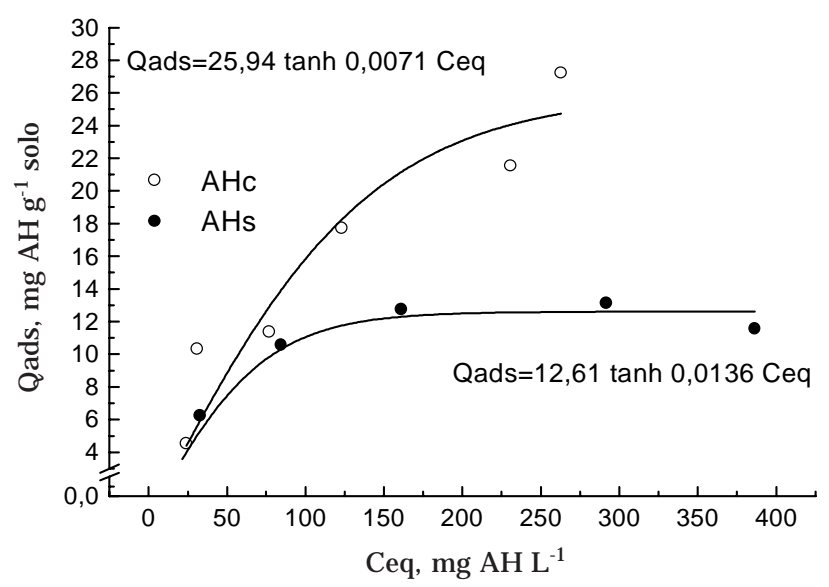

Figura 1. I sotermas de adsorção de áci dos húmicos extraídos de solo (AHs) e de carvão (AHc) em L atossolo Roxo natural. C eq = concentração de AH em equilíbrio e Qads = AH adsorvido. Os coeficientes de determinação das equações são apresentados no quadro 3.

Observa-se, no quadro 3 , que os coeficientes de determinação obtidos com o modelo de Langmuir foram relativamente altos, porém apenas no caso do AHc esta equação descreveu mais adequadamente a adsorção no LR natural do que a equação da tangente hiperbólica.

Considerando que o model o de Langmuir aplicase a adsorventes que apresentam al ta afinidade pel o adsorbato, princi palmente em baixas concentrações deste (Sparks, 1995), este resultado indica que a interação da molécula orgânica e a superfície dos minerais foi de natureza química. $\mathrm{Na}$ adsorção de matéria orgânica aquática em óxidos de ferro sintético, a isoterma de Langmuir descreveu adequadamente os dados experimentais obtidos por Gu et al. (1994). Estes autores observaram por meio deanálise por DRIFT e de ${ }^{13 C}$-RMN quea interação deveu-se, principalmente, à troca de ligantes entre a superfície e a molécula orgânica e que a natureza da reação foi extretamente forte e parcialmente reversível.

A quantidade máxima adsorvida do $\mathrm{AHC}$ (39,32 $\left.\mathrm{mg} \mathrm{AH} \mathrm{g}^{-1}\right)$, calculada segundo o modelo de Langmuir, foi aproximadamente 2,5 vezes superior à do $\mathrm{AHs}\left(15,51 \mathrm{mg} \mathrm{AH} \mathrm{g}^{-1}\right)$. A normalização deste parâmetro para unidade de área forneceu valores superiores (1,2 vez, para oAHs, e 1,5 vez para oAHc) aos obtidos com a equação da tangente hiperbólica (Quadro 3), porém confirmam a tendência observada com aquela equação de que o AHc é adsorvido em maiores quantidades do que o AHs. A amostra AHc difere da amostra AHs em razão do maior teor de grupamentos carboxílicos na sua estrutura (Quadro 2). Infere-se, portanto, que este grupo funcional participa da adsorção provavelmente por meio de reação de coordenaçãoo de superfície com os minerais. Adicionalmente, ambos os $\mathrm{AH}$ apresentaram em sua estrutura o predomínio de caráter aromático em relação ao alifático, sendo o fator aromaticidade no AHc $(0,76)$ superior ao do AHs $(0,60)$ (Quadro 2), evidenciando que estruturas aromáticas também contribuem para a adsorção. Recentemente, Kaiser et al. (1997) comprovaram, através de espectroscopia de DRIFT e 13C-RMN, a importância dos grupos carboxílicos e estruturas aromáticas na adsorção deácidos húmi cos em óxidos naturais e sintéticos. Wang et al. (1997) obtiveram, para Xmax na adsorção deáci do fúlvico em goethita sintética, cal culada segundo o model o de Langmuir,

Quadro 3. Área superficial específica (ASE); $\mathrm{pH}$ em $\mathrm{H}_{2} \mathrm{O}\left(\mathrm{pH}_{\mathrm{H} 2 \mathrm{O}}\right)$; coeficiente de determinação $\left(\mathrm{R}^{2}\right)$ e adsorção máxima, Xmax (por peso e por área de adsorvente) para o modelo linearizado de Langmuir; coeficiente de determi nação $\left(R^{2}\right)$, adsorção máxi ma, $A$ (por peso e por área de adsorvente), e produto A.B segundo a equação y = A tanh Bx; para Latossolo Roxo natural e Latossolo Roxo tratado com oxalato de amônio. * resultados sem significância estatística

\begin{tabular}{|c|c|c|c|c|c|c|c|c|c|}
\hline \multirow{2}{*}{ Adsorvente } & \multirow{2}{*}{ ASE } & \multirow{2}{*}{$\mathrm{pH}_{\mathrm{H}_{2} \mathrm{O}}$} & \multicolumn{3}{|c|}{ Langmuir } & \multicolumn{4}{|c|}{$\mathbf{Y}=\mathrm{A} \tanh \mathrm{Bx}$} \\
\hline & & & $\mathbf{R}^{2}$ & Xmax & Xmax & $\mathbf{R}^{2}$ & $\mathbf{A}$ & $\mathbf{A}$ & A.B. \\
\hline \multirow{3}{*}{$\begin{array}{l}\text { Latossolo } \\
\text { natural }\end{array}$} & $m^{2} g^{-1}$ & & & $\mathrm{mg} \mathrm{AH} \mathrm{g-1}$ & $\mathrm{mg} \mathrm{AH} \mathrm{m} \mathrm{m}^{-2}$ & & $\mathrm{mg} \mathrm{AH} \mathrm{g} \mathrm{g}^{-1}$ & $\mathrm{mg} \mathrm{AH} \mathrm{m} \mathrm{m}^{-2}$ & $\mathrm{~L} \mathrm{~m}^{-2}$ \\
\hline & 66,1 & 7,5 & AHs: 0,87 & AHs: 15,56 & AHs: 0,23 & AHs: 0,94 & AHs: 12,61 & AHs: 0,19 & AHs: 0,0026 \\
\hline & & & AHc: 0,84 & AHc: 39,31 & AHc: 0,43 & AHc: 0,80 & AHC: 25,94 & AHc: 0,39 & AHc: 0,0028 \\
\hline \multirow{2}{*}{$\begin{array}{l}\text { Latossolo } \\
\text { tratado }\end{array}$} & 140,9 & 5,5 & AHs: 0,50 & $*$ & * & AHs: 0,89 & AHs: 62,32 & AHs: 0,44 & AHs: 0,0186 \\
\hline & & & AHC: $9.10^{-4}$ & $*$ & $*$ & AHc: 0,86 & AHc: 122,38 & AHc: 0,87 & AHC: 0,0160 \\
\hline
\end{tabular}


um valor de 1,6 mg AF m-2, superior aos obtidos no presente estudo. (Quadro 3). Os testes de adsorção realizados por estes autores foram em $\mathrm{pH}$ 5,8, tendo a goethita sintética apresentado el evada carga de superfícielíquida positiva, oque, provavelmente, não acontece nos adsorbatos naturais empregados neste trabalho. Wang et al. (1997) constataram também, pelo fracionamento por peso molecular, que ácidos fúlvicos de maior cadeia, mais aromáticos e com maior proporção de grupamentos carboxílicos, foram adsorvidos preferencialmente em detrimento de moléculas de menor peso molecular e com menor teor de carboxilas nas suas estruturas.

Os valores calculados para a afinidade química (produto A.B, em L m-2), segundo a equação da tangentehiperbólica, mostram quea força deinteração da superfície dos minerais e o adsorbato foi semel hante para os dois AH estudados (Quadro 3).

\section{Adsorção em Latossolo Roxo tratado com oxalato de amônio}

As isotermas de adsorção obtidas para o solo tratado com oxal ato de amônio mostram que, para o $\mathrm{AHs}$, os valores de quantidade adsorvida tenderam para um nível de saturação, enquanto, para o $\mathrm{AHc}$, a adsorção apresentou variação praticamentelinear com o aumento da concentração de $\mathrm{AH}$ em equilíbrio na solução (Figuras 2a e 2b). A adsorção máxima estimada segundo a equaçãoy $=\mathrm{A}$ tanh $\mathrm{B} x$ foi aproximadamente 2 vezes mai or no $\mathrm{AHc}\left(122,38 \mathrm{mg} \mathrm{AH} \mathrm{g}^{-1}\right.$ solo) do que no AHs (62,32 $\mathrm{mg} \mathrm{AH} \mathrm{g}^{-1}$ solo), repetindo-se o comportamento observado no $L R$ natural (Quadro 3). Comparando os dois adsorventes, a capacidade adsortiva por unidade de área, A, do LR tratado foi 2,2 a 2,3 vezes superior à do LR natural.
O tratamento com oxalato de amônio, segundo Schwertmann (1964), extrai a fração pobremente cristalina dos óxidos de ferro, que apresenta maior reatividade de superfície do que a fração bem cristalizada. E m Latossol os, a proporção desta forma de ferro é geralmente muito baixa $\left(\mathrm{Fe}_{\mathrm{o}} / \mathrm{Fe}_{\mathrm{d}}<0,03\right)$ (Dick, 1993; Bayer, 1996). A pesar do baixo conteúdo de óxi dos de ferro pobremente cristalinos neste solo $\left(5,99 \mathrm{~g} \mathrm{~kg}^{-1}\right)$, esperava-se que, em virtude de sua alta reatividade, o tratamento com oxalato de amônio resultasse numa diminuição da adsorção dos $\mathrm{AH}$, o que nãose confirmou. A amostra tratada com oxalato deamônio apresentou a ASE mai or do quea amostra natural (Quadro 3). Provavelmente, o tratamento com oxalato extraiu a parte superficial dos cristais de óxidos de ferro, rompendo microagregados e liberando mais sítios reativos para a adsorção. 0 menor valor de $\mathrm{pH}$ do LR tratado, quando comparado com o LR natural (Quadro 3), pode também ter contribuído para a maior capacidade de adsorção, uma vez que em pH 5,5 a proporção de carga superficial positiva é maior do que em pH 7,5.

Contrariamente ao observado no LR natural, o modelo de Langmuir não descreveu adequadamente os resultados experimentais obtidos (Quadro 3), o que pode ser indicativo de que mecanismos diferentes deadsorção atuaram nos dois adsorventes. Por outro lado, considerando a maior capacidade adsortiva do LR tratado, as quantidades de $\mathrm{AH}$ em equilíbrio foram baixas, para que se obtivesse um patamar de saturação, inviabilizando a apli cação do model o de Langmuir.

A afinidade química (A.B) de cada adsorbato pela superfície do sol o tratado foi semel hante. Verificase, porém, que, no LR tratado, a afinidadeé deseis a sete vezes maior do que no LR natural (Quadro 3).

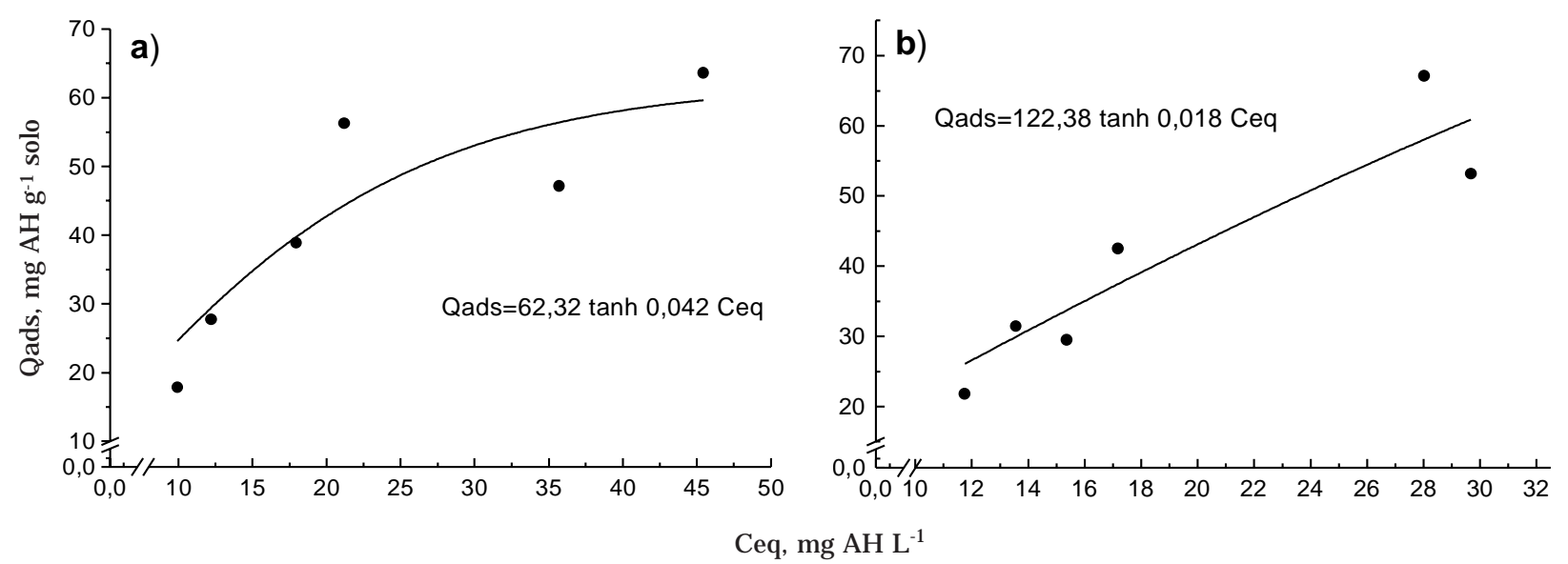

Figura 2. I sotermas de adsorção de áci dos húmi cos extraídos de a) solo e de b) carvão em Latossolo Roxo tratado com oxalato de amônio. Ceq = concentração de AH em equilíbrio e Qads = AH adsorvido. Os coeficientes de determi nação das equações são apresentados no quadro 3. 
Infere-se que as diferenças de afinidade foram governadas principal mente pelas características do adsorvente e não dos adsorbatos.

Os sítios expostos após o tratamento com oxalato de amônio poderiam ser mais reativos. Além disso, o oxalato adsorvido na amostra tratada, remanescente ao tratamento com $\mathrm{H}_{2} \mathrm{O}_{2}$, conforme mostram as análises de DRIFT (ver item mais adiante), podeter criado sítios de adsorção de maior afinidade química pelo $\mathrm{AH}$. A parte hidrofóbica do oxalato que se posiciona mais externamente, uma vez que as carboxilas estão coordenadas com a superfície (Parfitt et al., 1977), adsorveria a parte hidrofóbica da molécula de $\mathrm{AH}$.

Num estudo comparativo de adsorção entre matéria orgânica hidrofílica e matéria orgânica hidrofóbica em óxidos sintéticos e em solos, Kaiser \& Zech (1997b) observaram que a fração hidrofóbica apresentou maior afinidade química pela superfície de goethita do quea hidrofílica. Segundo os autores, tal comportamento pode ser devido ao maior peso molecular, ao maior teor de estruturas aromáticas e, ou, a um arranjo estérico favorável dos grupos funcionais da fração hidrofóbica. A importância do mecansimo de interação hidrofóbica na adsor ção de ácidos fúlvicos em goethita sintética foi também constatada por Wang et al. (1997).

\section{Análise por espectroscopia no infravermelho por reflectância difusa com transformada de fourier (DRIFT)}

O espectro do LR natural antes da adsorção (F igura 3a) foi composto por bandas de absorção dos grupos de superfície dos minerais: as bandas em 3.696, 3.659 e $3.622 \mathrm{~cm}^{-1}$ são devidas aos estiramentos dos grupos Al-OH eSi-OH da caulinita; a banda em $3.426 \mathrm{~cm}^{-1}$ corresponde ao estiramento dogrupo $\mathrm{OH}$ em ponte; a banda em $1.647 \mathrm{~cm}^{-1}$ édevida ao estiramento $\mathrm{OH}$ da água estrutural dos minerais; a banda larga em torno de $1.370 \mathrm{~cm}^{-1}$ é devida à absorção de grupos Al-O eF e-O eas bandas em 1.158 e $1.106 \mathrm{~cm}^{-1}$ são devidas à absorção de grupos Si-O.

No espectro de LR natural adsorvido com AHc (F igura 3b), foram identificadas as bandas em 2.961, 2.923 e $2.858 \mathrm{~cm}^{-1}$ correspondentes aos estiramentos de $\mathrm{C}-\mathrm{H}$ degrupos de $-\mathrm{CH}_{3} \mathrm{e}-\mathrm{CH}_{2}$. A banda em torno de $1.647 \mathrm{~cm}^{-1}$ no espectro a sofreu um alargamento no espectro b. 0 ombro em $1.705 \mathrm{~cm}^{-1}$ é atribuído ao estiramento de $\mathrm{C}=\mathrm{O}$ de grupos $\mathrm{COOH}$ eos ombros em 1.570 e $1.370 \mathrm{~cm}^{-1}$ podem ser devidos aos estiramentos simétricos e anti-simétricos, respectivamente, de $\mathrm{C}=\mathrm{O}$ de grupos COOfortemente ligados à superfície. O deslocamento da banda de absorção de carboxilato de $1.647 \mathrm{~cm}^{-1}$ para $1.570 \mathrm{~cm}-1$ é devido a interações quími cas fortes com metal, interações do tipo troca de ligantes e a superfície dos óxidos (Gu et al., 1994; Kaiser \& Zech, 1997a). A banda em $1.622 \mathrm{~cm}^{-1}$ pode ser tanto atribuída ao estiramento de $\mathrm{C}=\mathrm{O}$ dequinonas como ao estiramento de $\mathrm{C}=\mathrm{C}$ de anel aromático, evidenciando a presença de estrutura aromática ligada à superfície mineral.

O espectro do LR tratado com oxalato de amônio antes da adsorção ( $F$ igura 4a) é semelhante ao do LR natural (Figura 3a), diferindo apenas quanto à presença da banda em $1.321 \mathrm{~cm}^{-1}$, que foi atribuída ao oxalato adsorvido na superfície do mineral e que não foi totalmente removido pelo tratamento com peróxido (Figura 4a). Segundo Nakamoto (1978), o estiramento de $\mathrm{C}$-O e C-C do oxalato que ocorre em torno de $1.390 \mathrm{~cm}^{-1}$ desloca-se para número de ondas mais baixo ao coordenar com o Fe.

O espectro da amostra adsorvida apresenta as mesmas bandas de absorção da matéria orgânica (Figura 4b) já observadas no espectro do LR natural adsorvido, acusando a presença de estruturas aromáticas eal ifáticas e de grupos COO- adsorvidos. A presença do oxalato na superfície pode ter determinado maior especificidade da interação organomineral.

\section{LATOSSOLO ROXO NATURAL}

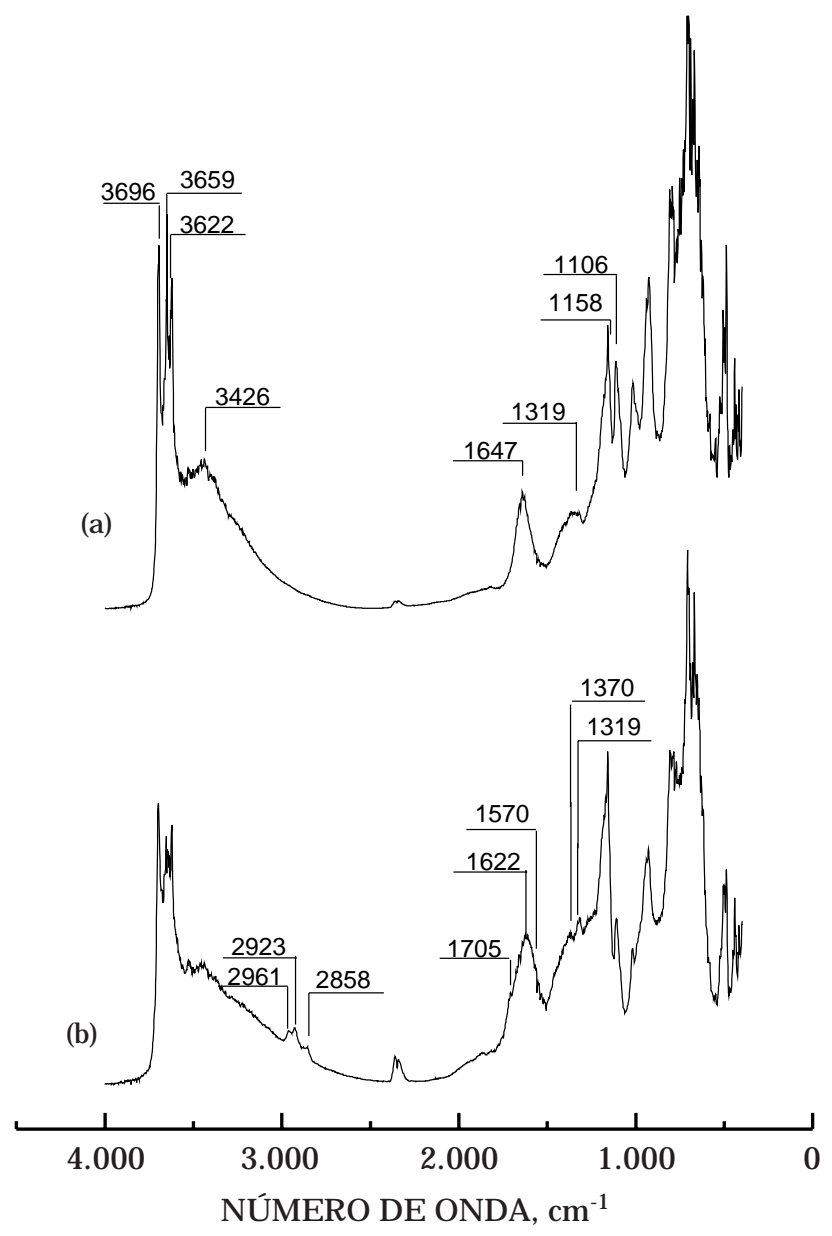

Figura 3. Espectro de DRIFT do Latossolo Roxo natural a) antes e b) depois da adsorção de ácido húmico extraído de carvão. 
LATOSSOLO ROXO NATURAL

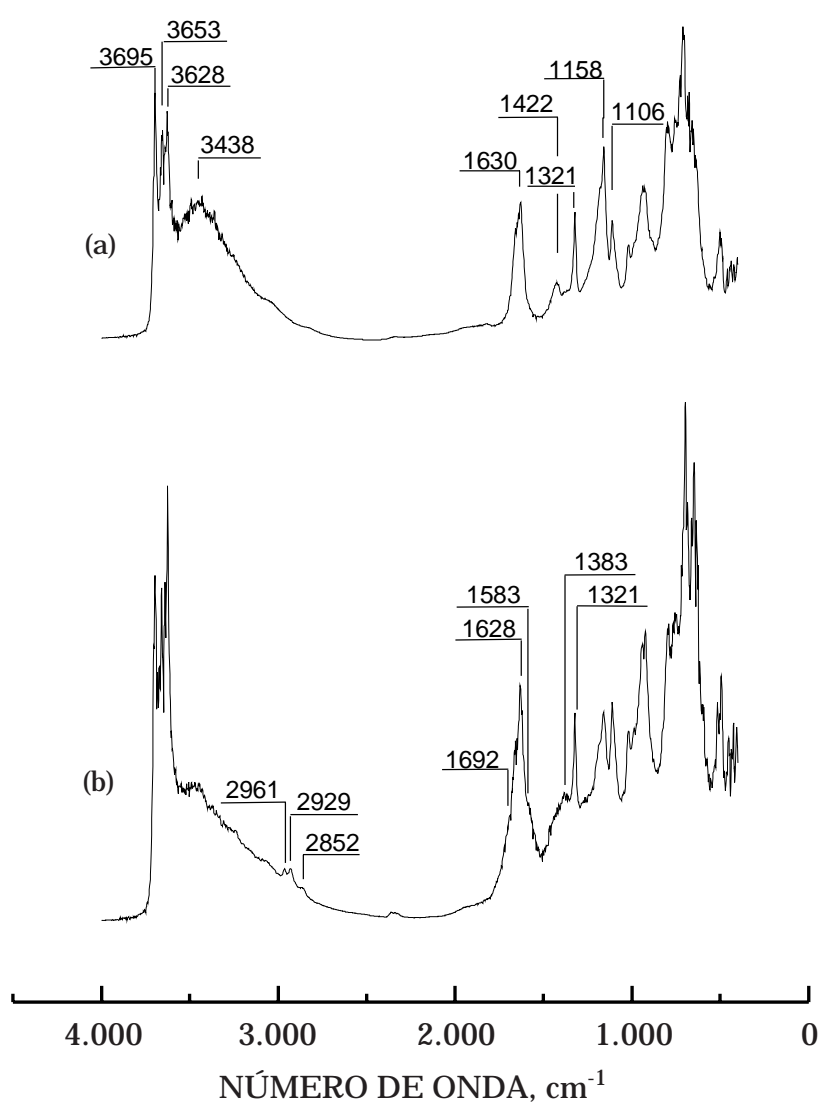

Figura 4. Espectro de DRIFT do LR tratado com oxalato de amônio a) antes e b) depois da adsorção de ácido húmico extraído de carvão.

Os espectros das amostras adsorvidas com $\mathrm{AHs}$ foram semel hantes aos obtidos com AHce, portanto, não foram apresentados.

\section{CONCLUSÕES}

1. O ácido húmico de carvão foi adsorvido em maior quantidadedo que oácido húmico de solo. Este comportamento foi relacionado com o caráter aromático mais el evado e com o maior teor de grupos carboxílicos da amostra de carvão.

2. A adsorção de ácidos húmicos em LR natural e em LR tratado foi de natureza química, parecendo ser a reação de trocas deligantes das carboxilas com a superfíciedos mi nerais um mecanismoimportante. Também foi determinada a presença de estruturas aromáticas no composto adsorvido nas superfícies minerais.

3. Em geral, a parametrização $y=A$ tanh $B x$ descreveu os dados experimentaismais adequadamente do que o modelo de Langmuir, apresentando sempre
$R^{2} \geq 0,80$. Esta equação permitiu o cálculo da adsorção máxima que, em três casos, coincidiu com os valores obtidos experimental mente. Adicional mente, a equação proposta permitiu avaliar relativamente a afinidade entre adsorbatos e adsorventes, o que é questionável no model o de Langmuir. Ainda que não tenha uma fundamentoteórico, esta parametrização apresenta-se como alternativa para a descrição analítica de adsorção de substâncias húmicas em superfícies de carga variável.

4. No LR tratado com oxalato de amônio, a adsorção por unidade de área foi mais elevada do que no LR natural, o que foi relacionado com a criação de maior número de sítios reativos após tratamento com o oxalato e menor nível de pH no primeiro adsorvente.

5. A técnica de espectroscopia de DRIFT permitiu identificar a presença de grupos carboxilatos na interação $\mathrm{AH}$-solo, sugerindo o mecanismo de troca de ligantes na adsorção.

\section{AGRADECIMENTOS}

Os autores agradecem ao Dr. H. Herzog doISAS, Dortmund, Alemanha, pelo auxílio na realização das análises de RMN-13C, e ao CNPq, FAPERGS e PROPESQ/UFRGS.

\section{LITE RATURA CITADA}

BAES, A.U. \& BLOOM, P.R. Diffusereflectanceand transmission fourier transform infrared (DRIFT) spectrocopy of humic and fulvic acids. Soil Sci. Soc. Am. J ., 53:695-700, 1989.

BAYER, C. Dinâmica da matéria orgânica em sistemas conservacionistas de manejo de solos. Porto Alegre, Universidade Federal do Rio Grande do Sul, 1996. 241p. (Tese de Doutorado)

DELINEAU, T.; ALIARD, T.; MULLER,J .P.; BARRES, O.; YVON, J. \& CASES, J .M. FTIR reflectance vs. EPR studies of structural iron in kaolinites. Clays Clay Miner., 42:308-320, 1994.

DICK, D.P. Caracterização deóxidos deferroeadsorção defósforo na fração argila de horizontes B-latossólicos. Porto Alegre, Universidade Federal do Rio Grande do Sul, 1986. 196p. (Tese de Mestrado)

DICK, D.P. Eigenschaften von Mikroaggregaten in brasilianischen Oxisolen. Freising, TechnischeUniversität München, 1993. 149p.

DICK,.D.P.; GOMES, J . \& ROSINHA, P.B. Caracterização de substâncias húmicas extraídas de solos e de lodo orgânico. R. Bras. Ci. Solo, 22:603-611, 1998.

DUXBURY, J .M.; SMITH, M.S. \& DORAN, J .M. Soil organic matter as a source and a sink of plant nutrients. In: COLEMAN, D.C.; OADES, J .M. \& UEHARA, G. Dynamics of soil organic matter in tropical ecossystems. Honolulu, University of Hawaii, 1989. p.33-67. 
EMPRESA BRASILEIRA DE PESQUISA AGROPECUÁRIA EMBRAPA. Centro Nacional de Pesquisa em Solos. Manual de métodos de análise de solo. 2.ed. Rio de janeiro, 1997. p.83-84.

FARMER, V.C. The infra-red spectra of minerals. London, Mineralogical Society, 1974. 537p. (Monograph, 4)

FONTES, M.R. Interactions of goethite and humic acid in some oxisols from Brazil. Raleigh, North Caroline State University, 1990. 75p. (Tese de Doutorado)

GU, B.; SCHMITT, J .; CHEN, Z.; LLANG, L. \& MaCCARTHY, J .F. Adsorption and desorption of natural organic matter on iron oxide: Mechanism and models. Environ.Sci. Technol., 28:38-46, 1994.

HATCHER, P.G.; SCHNITZER, M.; DENNIS, L.W. \& MACIEL, G.E. Aromaticity of humic substances. Soil Sci. Soc. Am. J ., 45:1089-1094, 1981.

KAISER, K. \& ZECH, W. Natural organic matter sorption on different mineral surfaces studied by DRIFT spectroscopy. Sciences of Soils, rel.2, 1997a. (http: hintze-online.com/sos/ 1997a/Articles/Art4)

KAISER, K. \& ZECH, W. Competitive sorption of dissolved organic matter fractions to soils and related mineral phases. Soil Sci. Soc. Am. J ., 61:64-69, 1997b.

KAISER, K.; GUGGENBERGER, G.; HAUMAIER, L. \& ZECH, $W$. Dissolved organic matter sorption on subsoils and minerals studied by 13 C-NMR and DRIFT spectroscopy. Eur. J . Soil Sci., 48:301-310, 1997.

MORELLI, M. \& BORTOLLUZZI, G. Efeito do humato e poligalacturonato na sorção de fosfato pelo solo. R. Bras. Ci. Solo, 16:373-377, 1992.

MUNEER, M. \& OADES, J .M. Therole of Ca-organic interactions in soil aggregatestability. III. Mechanisms and models. Aust. J . Soil Res., 27:411-423, 1989.

NAKAMOTO, K. Infrared and raman spectra of inorganic and coordination compounds. 3 .ed. London, Chapmann \& Hall, 1978. p.234-235.

OLSEN, S.R. \& WATANABE, F.S. A method to determine a phosphorus adsorption maximum of soil as measured by the Langmuir isotherm. Soil Sci. Soc. Am. Proc., 21:144149, 1957.
PARFITT, R.L.; FRASER, A.R. \& FARMER, V.C. Adsorption on hydrous oxides. III. Fulvic acid and humic acid on goethite, gibbsite and imogolite. J . Soil Sci., 28:289-296, 1977.

QUIRK, J .P. Significance of surface areas calculated from water vapour sorption isotherms by the use of the BET equation. Soil Sci., 80:423-430, 1955.

RISTORI, G.G.; SPARVOLLI, E.; NOBILI, M. \& D'ACQUI, L.P. Characterization of organic matter in particle-size fractions of vertisols. Geoderma, 54:295-305, 1992.

SCHULTHESS, C.P. \& HUANG, C.P. Humic and fulvic acid adsorption by silicon and aluminum oxide surfaces on clay minerals. Soil Sci. Soc. Am. J ., 55:34-42, 1991.

SCHWERTMANN, U. Differenzierung der Eisenoxidedes Bodens durch Extraktion mit Ammonium-Oxalat Lösung. Z. Pflanzenernahr. Bodenkd. 105:194-202, 1964.

SKJ EMSTAD, J .O.; CLARK, P.; GOLCHIN, A. \& OADES, J .M. Characterization of soil organic matter by solid-state ${ }^{13} \mathrm{C}$ NMR spectroscopy. In: CADISH, G. \& GILLER, K., eds. Driven by nature: Plant litter quality and decomposition. Wallingford, CAB International, 1996. p.372.

SPARKS, D. Environmental soil chemistry. San Diego, Academic Press, 1995. 267p.

SPOSITO, G. The chemistry of soils. New York, Oxford University Press, 1989. 277p.

STEVENSON, F.J . Humus chemistry. Genesis, composition, reactions. 2.ed. New York, J ohn Wiley, 1994. 496p.

TEDESCO, M.; GIANELLO, C.; BISANI, C.A.; BOHNEN, H. \& VOLSWEISS, S. Análise de solo, plantas e outros materiais. 2.ed. Porto Alegre, Universidade Federal do Rio Grande do Sul, 1995, 174p. (Boletim Técnico, 5)

TIPPING, E. The adsorption of aquatic humic substances by iron oxides. Geochim. Cosmochim. Acta, 45:191-199, 1981.

VALLADARES, G.S.; PEREIRA, M.G. \& ALVES, G.C. Aplicação de duas isotermas de adsorção de boro em sol os de baixada do estado do Rio de J aneiro. R. Bras. Ci. Solo, 22:361-365, 1998.

VARADACHARI, C.; CHATTOPADHYAY, T. \& GHOSH, K. Complexation of humic substances with oxides of iron and aluminum. Soil Sci., 162:28-34, 1997.

WANG, L.; CHIN, Y.P. \& TRAINA, S.J . Adsorption of (poly)maleic acid and aquatic fulvic acid by goethite. Geochim. Chosmochim. Acta, 61:5313-5324, 1997. 